\title{
Quantum technology with diamond NV centers
}

H. Bernien, B. Hensen, W. Pfaff, G. Koolstra, M. S. Blok, L. Robledo, T. H. Taminiau, M. Markham, D. L. Twitchen, L. Childress, and R. Hanson, "Heralded entanglement between solid-state quits separated by three metres," Nature 497, 86-90 (2013).

\section{Recommended with a Commentary by David DiVincenzo, RWTH Aachen}

Among the hundreds of known color centers, the nitrogen-vacancy complex in diamond, in its -1 charge state, has almost alone become the focus of intense experimental investigation for the implementation of qubits, or more generally for the implementation of quantum assisted technologies like magnetometry and thermometry. It is not clear that this was inevitable, but given the massive knowledge built up about NV in the last few years, it would probably be impossible to achieve the feat of separated entanglement, as reported in this paper from Hanson's laboratory, in any other solid state system.

There are definitely features of the $\mathrm{NV}^{-}$that make it a good choice. Its ground state has a finite electron spin $(\mathrm{S}=1)$, providing a convenient, high-coherence set of states. Their splittings (at zero magnetic field it is $2.88 \mathrm{GHz}$ ) are optimal for easy microwave manipulation. Optical properties are also straightforward, simple, and useful; resonant and non-resonant excitation are with "red" and "green" lasers, i.e., in the easiest range for the laser scientist. The spin environment, at least, is clean: the nitrogen nuclear spin is easy to keep out of the physics, if necessary, and the carbons are mostly spin free. The occasional spin- $1 / 2$ C-13 nucleus is actually a very useful additional tool in the qubit toolkit, as electronic spin coherence can be swapped into the C-13 nucleus, making it very stable and long lived.

There are definitely features of the $\mathrm{NV}^{-}$that make it a bad choice. Dealing with the

badness is most of the formidable technical achievement of Bernien et al. In the experiment, they need two identical copies of an NV emitter (separated by three meters). "Emitter" means something specific: upon emission, a single photon of a specific wavelength and spatial mode should be entangled with the NV spin ground state. The list of things that go wrong is quite long: 1) Usually (97\% of the time) emission also comes along with a phonon - these events are recognised and discarded. 2) Usually the photon goes in the wrong direction. This 
is dealt with using ingenious previous inventions of solid lenses sculpted into the diamond around the specific NV of interest. 3) NVs are very sensitive to strain and Stark fields, so they are not identical. Additional Stark fields are applied to make them identical. 4) The NV centre gets occasionally phtoionized, requiring occasional repumping to bring it back - but the repumpling sometimes causes jumps in the electrostatic environment, requiring further retuning. 5) Photons get lost on their $3 \mathrm{~m}$ transit to the entangling joint detection; a double repetition protocol insensitive to loss was invented.

There are about four additional tricks invented in this paper to make entanglement more likely. With all this, successful entanglement takes place about one time in ten million, at a rate of about one every 10 minutes; 739 entangling events occurred in about a week of running. Our particle physics friends will appreciate the care with which this experiment was done, with the very recognizable goal to get the count rate just large enough for the physics to be seen. When successful, the entangling event is impressive: two spins, in two separate crystals $3 \mathrm{~m}$ apart, remain in a high-fidelity entangled state for about $10 \mathrm{msec}$. To put this in perspective, in previous experiments where entangled photons are created and fly into free space, this time corresponds to a separation distance of $6000 \mathrm{~km}$ - definitely beyond what anyone can imagine doing. By swapping to C-13 spins (hoped for in this paper, but not yet done), the storage lifetime will be 100 times longer, long enough for entangled photons to take a trip to the moon.

A final thought about this experiment: since its elucidation 75 years ago[1], we have considered $1 /$ f noise as an unavoidable and uncontrollable feature of solid state devices. The control tricks of this paper show what it takes, finally, to completely tame this noise. An ensemble of NVs are a good example of a sea of $1 / \mathrm{f}$ fluctuators. At the cost of fanatical, intrusive, and constant manipulation at the single-impurity level, fluctuators can in fact be tamed.

[1] J. Bernamont, "Fluctuations de potentiel aux bornes dún conducteur metallique de faible volume parcouru par un courant," Annales de Physique 7, 71 (1937). 